\title{
Hybrid Genetic Algorithm for Electromagnetic Topology Optimization
}

\author{
Chang-Hwan Im, Hyun-Kyo Jung, Senior Member, IEEE, and Yong-Joo Kim
}

\begin{abstract}
This paper proposes a hybrid genetic algorithm (GA) for electromagnetic topology optimization. A two-dimensional (2-D) encoding technique, which considers the geometrical topology, is first applied to electromagnetics. Then, a 2-D geographic crossover is used as the crossover operator. A novel local optimization algorithm, called the on/off sensitivity method, hybridized with the 2-D encoded GA, improves the convergence characteristics. The algorithm was verified by applying it to various case studies, and the results are presented herein.
\end{abstract}

Index Terms-Genetic algorithm (GA), geographic crossover, local optimization, topology optimization, two-dimensional (2-D) encoding.

\section{INTRODUCTION}

$\mathbf{S}$ TOCHASTIC optimization algorithms have been widely used for various electromagnetic optimization problems, in particular to optimize the geometrical dimensions of various electromagnetic devices [1]-[4]. The genetic algorithm (GA), simulated annealing (SA), evolution strategy (ES), and tabu search algorithm, etc. are all well-known examples of the stochastic algorithms. Unfortunately, they have not been applied much to direct shape optimization or topology optimization problems [5], because they carry a severe computational cost and have difficulty in dealing with large numbers of design variables. Instead, sensitivity analysis has attracted much interest because it can deal with large amounts of design variables quickly and effectively [6]-[8]. However, it has the problem that a solution may converge on a local minimum, due to its natural deterministic characteristic that stems from the derivatives of the objective functions.

In this paper, a hybrid GA for electromagnetic topology optimization is proposed. The GA adopts a two-dimensional (2-D) encoding [9] to represent the geometrical topology effectively. A multidimensional geographic crossover is applied to increase the diversity of the population [10]-[12]. The effectiveness of this approach has already been verified through its application to computer science [10], [11] and very large scale integration (VLSI) circuit design [12]. However, its application to topology optimization is totally new, not only in electromagnetic optimizations, but also in mechanical ones.

In this paper, to improve the accuracy and effectiveness of the "conventional" GA, a novel local optimization algorithm called the on/off sensitivity method is proposed and hybridized with a 2-D encoded GA. The proposed local optimization method

Manuscript received July 23, 2002; revised May 19, 2003.

C.-H. Im and H.-K. Jung are with the School of Electrical Engineering and Computer Science, Seoul National University, Seoul 151-742, Korea (e-mail: ichich2@snu.ac.kr; hkjung@snu.ac.kr).

Y.-J. Kim is with the Korea Electrotechnology Research Institute (KERI), Kyungsangnam-do 641-120, Korea (e-mail: yjkim@keri.re.kr).

Digital Object Identifier 10.1109/TMAG.2003.817094

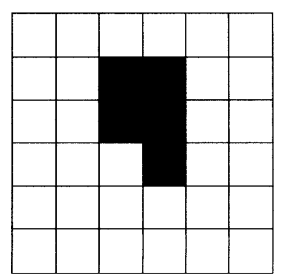

(a)

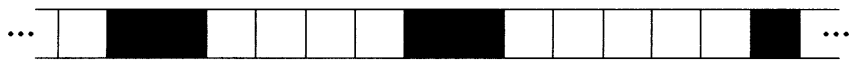

(b)

Fig. 1. Comparison of 1-D and 2-D encoding from the viewpoint of schemata. (a) 2-D encoding and its schemata. (b) Equivalent 1-D encoding and its schemata. (b) is a 1-D representation of (a). We can see from the figures that the 1-D encoding may lose its neighboring information, as shown in (b).

assists the main GA to generate superior solutions, and at the same time, the main GA perturbs the local solutions and prevents them from converging to a local minimum too prematurely. An appropriate combination of the two algorithms can accelerate the convergence speed and minimize the possibility of being trapped in a local optimum.

To verify the proposed algorithm, three case studies were considered, including a simplified magnetoencephalography (MEG) source localization problem, a current source optimization problem, and a brushless DC motor (BLDCM) optimization for reduced cogging torque. Through the case studies, it will be shown that the proposed method can yield reasonable solutions with high efficiency.

\section{MEthodOLOGY}

\section{A. 2-D Encoding for $G A$}

A linear string is a symbolic feature of GAs, and most GA implementations have been based on linear encodings [13]. To fit into linear strings, solutions are encoded into one-dimensional (1-D) chromosomes, as shown in Fig. 1(b). However, if 2-D geometry is embedded into a 1-D array, considerable distortion of neighboring information is unavoidable, as we can see from Fig. 1(a) and (b). Due to the broken schemata, the 1-D encoding has always suffered from its poor convergence characteristic.

Cohoon and Paris first proposed 2-D encodings and demonstrated moderate success in solving such a problem [9]. This approach led to the development of various crossover methods, which will be described in Section II-B.

\section{B. 2-D Crossover Methods}

Crossover is the most representative and important feature of GAs, which is also true of 2-D GAs. Cohoon and Paris, who first proposed the 2-D encoding, used a rectangular crossover 


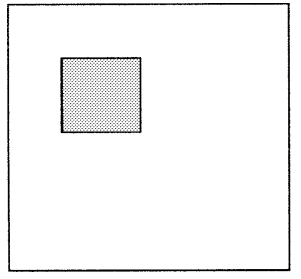

(a)

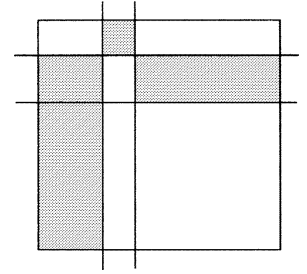

(b)

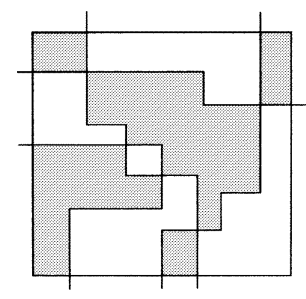

(c)
Fig. 2. Crossover methods. (a) Cohon and Paris-rectangular boxes were used. (b) Anderson - parallel lines were used. (c) Bui and Moon-more natural lines were used. The white region represents genes from parent 1 and the gray region represents those from parent 2 . The recent method (c) can generate more diverse solutions than the others.

[9]. In their approach, given two parent chromosomes, the algorithm chooses a small rectangle from one parent and copies the genes in the rectangle into the offspring; the remainder of the genes are copied from the other parent, as shown in Fig. 2(a). Anderson et al. suggested a block-uniform crossover on a 2-D matrix chromosome that tessellated the chromosome into $i \times j$ blocks; for each block, the genes in the block are copied from a uniformly selected parent, as shown in Fig. 2(b) [14]. It is evident that the block uniform crossover has a greater opportunity to generate more diverse offspring.

Most recently, Bui and Moon proposed a geographic crossover to increase the diversity of offspring [10]. It generalized the conventional block uniform crossover and introduced natural lines, as shown in Fig. 2(c). It was proven that the whole encoding space could always be divided into two separated regions, as shown in the figure. The offspring can be generated by alternatively copying the intervals of two parent strings. It was also proven that the geographic crossover could generate more diverse offspring than any of the conventional crossovers [10]-[12]. Because it is somewhat difficult to implement the method practically, we will introduce a relatively simple process used in this paper.

Step 1) First, one should bear in mind that there can be six possible cutting lines: a) top-to-right; b) top-toleft; c) bottom-to-left; d) bottom-to-right; e) top-tobottom; and f) left-to-right. The whole space is separated into two regions by one cutting line. Then, one should define a rule to assign 0 or 1 to each region, for all cases a)-f). Fig. 3(a)-(d) shows the examples of $0 / 1$ assignment.

Step 2) After generating all the cutting lines, the assigned numbers are summed up for every gene as in Fig. 3(e).

Step 3) If the summed number has an odd value, the offspring copies the gene from parent 1 , and vice versa. This process is applied to all genes. Then, as shown in Fig. 3(f), we can see that the whole space is separated into two regions very effectively.

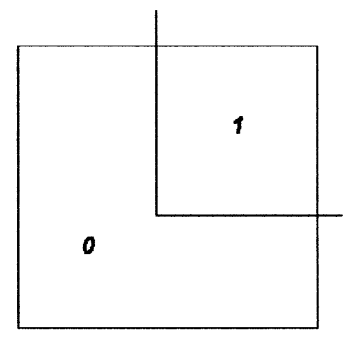

(a)

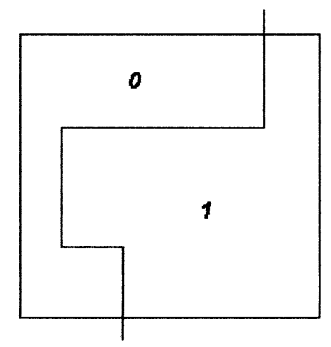

(c)

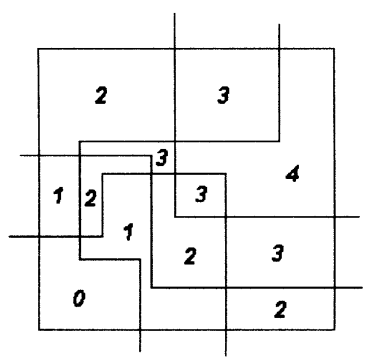

(e)

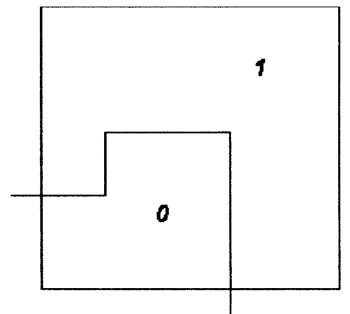

(b)

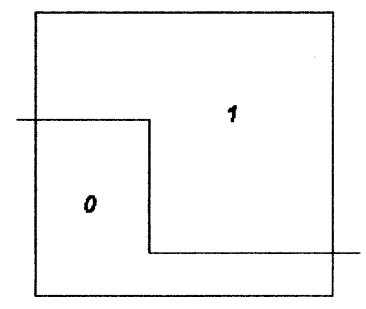

(d)

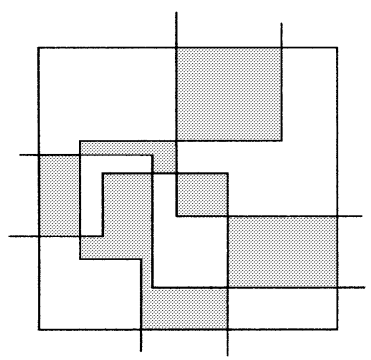

(f)
Fig. 3. Example of regional separation process: (a)-(d) show examples of basic cutting lines and rules for assigning region number. The assigned numbers for each region are summed up as shown in (c). Then, the whole region can be separable into two groups by checking if the summed numbers are odd or even, as shown in (f).

\section{New Local Optimization Algorithm-On/Off Sensitivity}

In this paper, a new local optimization algorithm for electromagnetic topology optimization is proposed. In essence, electromagnetic topology optimizations optimize the distribution of materials such as current sources, magnetic materials, etc. Hence, binary encoding is appropriate to represent whether such materials are present or not. In this paper, 1 represents the presence of a material and 0 represents the absence of it. The proposed local optimization algorithm adopts the concepts of both a sensitivity analysis and SA. The algorithm procedure is as follows.

Step 1) Calculation of On/Off Sensitivity. Change the state of each gene-if the state is 0 (off), replace it by 1 (on), and vice versa. Then, check the variation (sensitivity) of cost value. Higher sensitivity of a gene implies that the change of state can improve the cost value much more.

Step 2) Change State of Genes. After calculating the sensitivity of all genes, change the state of some genes, selectively. More sensitive genes are selected for 
mutation and the number of genes selected for mutation is defined as follows:

$$
N_{c}=N_{i} \times P
$$

where $N_{c}$ is the number of genes that change their states $(0 \rightarrow 1,1 \rightarrow 0), N_{c}$ is the number of genes that have positive sensitivity values, and $P$ is a probability between 0 and 1 , which will be explained again at the end of this section.

Step 3) Annealing. Check the cost value after the mutation. If the cost value is not improved at all, decrease the value of $P$ using the following annealing scheme:

$$
P(\text { new })=P(\text { old }) \cdot 0.85 \text {. }
$$

Any value can be used instead of 0.85 . In this paper, 0.85 is selected because it is usually used for conventional annealing processes. Repeat Step 2)-Step 3) until some improvement of the cost value is detected.

Step 4) Repeat Step 1)-Step 3) until the cost value is not improved any more.

Fig. 4 shows an example of the process of the local optimization algorithm. Assume that Fig. 4(a) is an optimal solution and Fig. 4(b) is an initial one. The calculated sensitivity profile is shown in Fig. 4(c). The positive sensitivity implies that cost value is improved when a gene changed its state, and vice versa. If the most sensitive gene changes its state, as shown in Fig. 4(d), it is evident that the cost value is improved. However, it seems somewhat inefficient because the cost value can be more improved by changing two or more genes' states. That is why we adopted a concept from SA. If the probability $P$ in (1) is set to be 1, the solution is changed, as shown in Fig. 4(e). From several simulations, it was observed that the cost value usually becomes worse (not improved at all) when too large numbers of genes are changed, as shown in Fig. 4(e). When the probability $P$ is decreased using (2), an improved solution can be obtained, as shown in Fig. 4(f). Then, the sensitivity computation is performed again, of which the result is shown in Fig. 4(g). From the sensitivity profile, we can find the optimal solution as shown in Fig. 4(h). In Step 2), the initial $P$ should be a large value close to 1 because there is no process to increase the probability. The initial $P$ is fixed throughout the whole iteration.

The proposed local optimization algorithm sometimes yielded very good characteristics, even without GA. In most cases, however, it converged to a local optimum, unless a good approximation of the initial solution was given.

\section{Whole Procedure of the Hybrid GA}

Usually, there are two main classes in hybrid GAs: Lamarckian and Baldwinian GAs. The Lamarckian GAs update the chromosome after the local optimizations, whereas the Baldwinian GAs do not update the chromosome after the local optimizations, and the local optimizations are only used as a fitness evaluation. Generally, the Lamarckian GAs have been more widely accepted and the Baldwinian GAs' use has been somewhat restricted [15], [16]. In this paper, we adopted the concepts of the Lamarckian GAs.

The proposed local optimization algorithm requires many function calls and consumes extensive computational time.

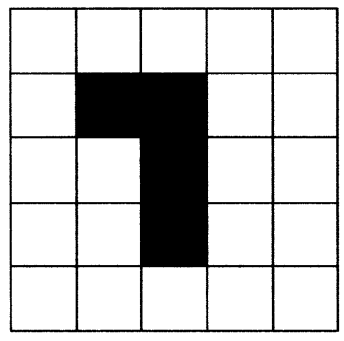

(a)

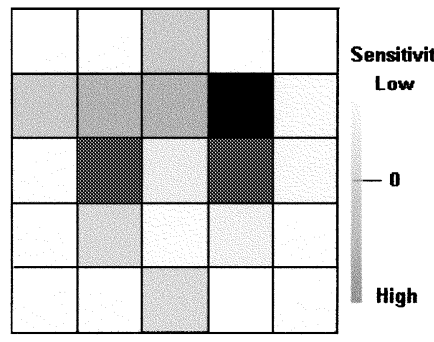

(c)

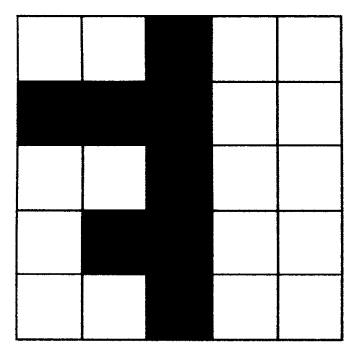

(e)

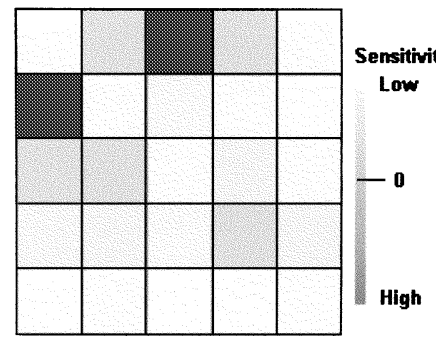

(g)

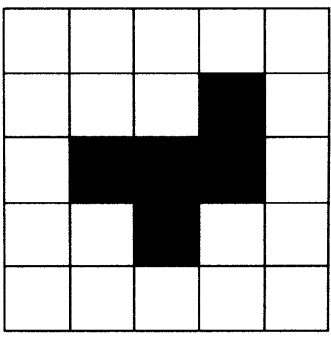

(b)

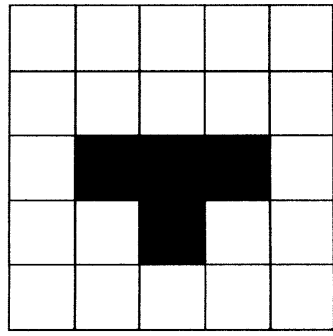

(d)

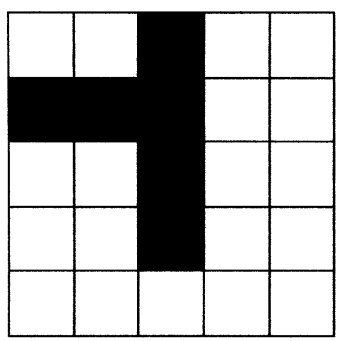

(f)

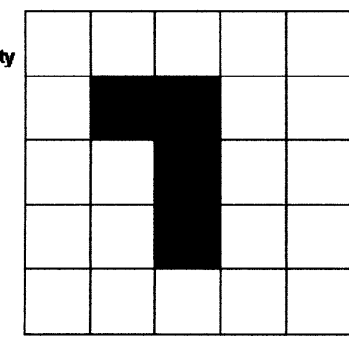

(h)
Fig. 4. An example to illustrate the process of the proposed local optimization algorithm (see text).

Moreover, excessive use of local optimization can degrade the diversity of the population. Therefore, in this paper, the proposed local optimization algorithm was not applied to every iteration or population. If a random number generated between 0 and 1 is larger than a predetermined probability, the on/off sensitivity is applied to some superior population. Fig. 5 shows the whole procedure of the proposed GA. In the procedure, a general mutation method is applied only when a random number between 0 and 1 is smaller than a predetermined probability, which perturbs a small fraction of the offspring. The predetermined probability of 0.015 is generally used; however, in this paper, larger values are used to alleviate premature convergence due to local optimization. After the mutation or crossover, worst solutions in the original population are replaced with newly generated ones. The GA procedure is terminated when $80 \%$ of the solutions have the same value. 


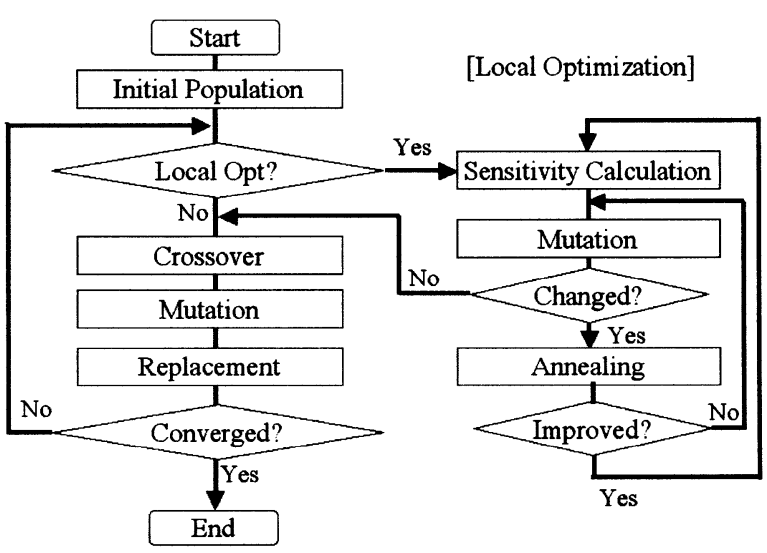

Fig. 5. Flowchart of the hybrid GA.

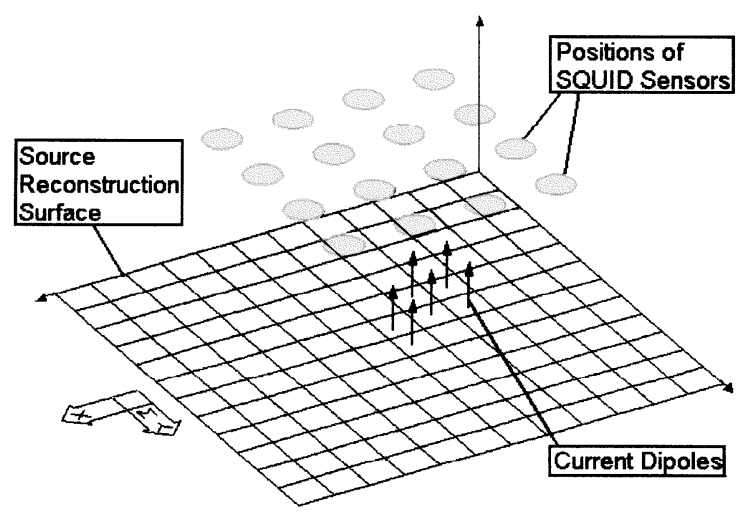

Fig. 6. Description of simulation model. Case study I: MEG source reconstruction.

\section{Simulation AND RESUlts}

The proposed algorithm was applied to three case studies: 1) a MEG source localization problem; 2) a current source optimization problem; and 3) a BLDCM optimization for reduced cogging torque.

\section{A. Case Study I-MEG Source Localization Problem}

Reconstructing electric activity inside a brain using magnetic measurements outside the head has attracted a great deal of interest and is usually referred to as the MEG source reconstruction problem [17], [18]. A simplified MEG model was selected for the verification of the optimization algorithm. Fig. 6 shows the schematic view of the simulation model. Sixteen $x / y$-directional superconducting quantum interference device (SQUID) magnetometers, capable of measuring parallel magnetic fields, were assumed. The magnitudes of the current dipole moments at $144(12 \times 12)$ rectangular meshes needed to be reconstructed. The magnitudes of the moments were fixed at a constant value and their directions were perpendicular to the source surface. Fig. 7 shows the exact (forward) solution that should have been reconstructed.

The main reason in selecting this model was that the forward solutions could be easily obtained using an analytic approach. ${ }^{1}$

\footnotetext{
${ }^{1}$ Some kinds of reconstruction problems such as a crack detection problem (materials to be reconstructed: air and conductor) can be classified as a topology optimization problem. Generally, the MEG source reconstruction problem is not such a problem, because the magnitude of each dipole cannot be determined a priori. Therefore, we simplified the problem to verify the optimization algorithm.
}

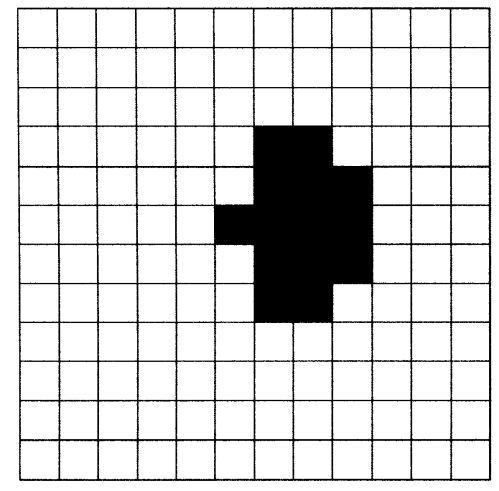

Fig. 7. Exact (forward) solution that is to be reconstructed.

TABLE I

CONDITIONS FOR EXECUTING GA

\begin{tabular}{cc}
\hline Population size & 3000 \\
Crossover frequency & 2000 (out of 3000) \\
Critical probability for mutation & 0.03 \\
Number of cutting lines & 7 \\
\hline
\end{tabular}

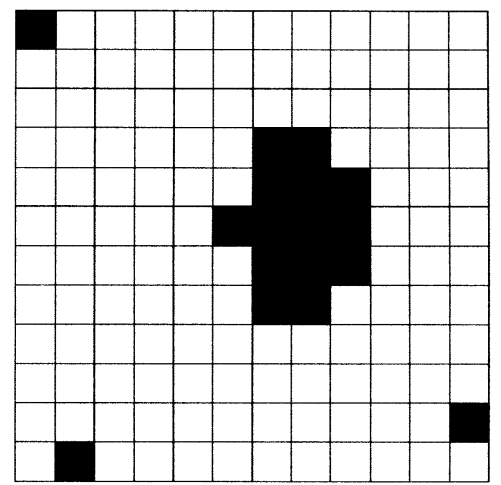

Fig. 8. Best reconstructed solution after 20 repeated executions of GA without local optimization.

The magnetic field induced by a current dipole moment can be calculated using Biot-Savart's law

$$
\boldsymbol{B}=\frac{\mu_{0}}{4 \pi} \frac{\boldsymbol{Q} \times\left(\boldsymbol{r}-\boldsymbol{r}_{\mathcal{Q}}\right)}{\left|\boldsymbol{r}-\boldsymbol{r}_{\mathcal{Q}}\right|^{3}}
$$

where $Q$ is the current dipole moment vector, $\boldsymbol{r}$ is the positional vector of the sensor, $\boldsymbol{r}_{\mathcal{Q}}$ is the positional vector of the dipole, and $B$ is the measured magnetic field at the sensor.

To apply GA, the objective function (cost) is defined to minimize the difference between the measured (forward) and reconstructed magnetic field at each sensor position.

Although the problem may seem very simple, GA has hardly been applied to such a problem because the total number of possible cases is so high; in this case $2^{144}$. For this reason, a general GA using a 1-D linear array and a general GA using 12 separated arrays failed to find the exact solution. However, the GA using a 2-D geographic crossover did succeed in finding a very close solution-of course, it was not exact. Table I shows the conditions required to execute the GA without local optimization. Fig. 8 shows the best reconstructed solution after 20 repeated applications of the GA without local optimization. 


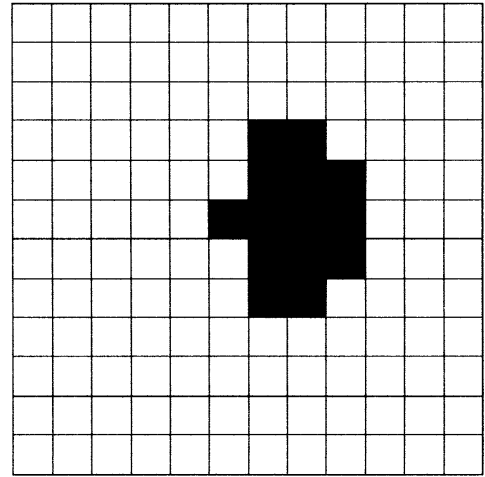

Fig. 9. Best reconstructed solution after 20 repeated executions of GA with local optimization.

TABLE II

COMPARISONS OF CAPABILITY OF GAS WITH AND WITHOUT LOCAL OPTIMIZATION ALGORITHM AFTER 20 REPEATED EXECUTIONS (CASE I)

\begin{tabular}{ccc}
\hline & Without Local Opt & With Local Opt. \\
\hline Best Solution $\left[\times 10^{-12} \mathrm{~T}\right]$ & 1.2 & 0.0 \\
Average Solution $\left[\times 10^{-12} \mathrm{~T}\right]$ & 8.8 & 2.1 \\
Worst Solution $\left[\times 10^{-12} \mathrm{~T}\right]$ & 19.7 & 4.3 \\
\hline
\end{tabular}

Because the objective function is defined as the difference between the measured and reconstructed magnetic field at each sensor position, smaller value represents better solution.

All conditions, which were applied to the proposed GA using local optimization, are given in Table I. The local optimization started to work after the 50th GA iteration; not from the start, because impatient use of the local optimization may have been harmful to the creation of the schemata. If a random number between 0 and 1 was larger than 0.8 , then local optimization was applied to 100 superior solutions. Fig. 9 shows the best reconstructed solution after 20 repeated applications of the GA with local optimization.

To verify the results quantitatively, best, worst, and average values of the objective function were compared. The results are given in Table II. From the table, the superiority of the GA with local optimization can be easily confirmed.

\section{B. Case Study II-Source Optimization Problem}

Magnetic resonance imaging (MRI) coil design is a typical application of the source optimization problem [19], [20]. Fig. 10 shows the numerical model under consideration and the initial magnetic field distribution. The problem was to make the $y$-directional flux density in a measuring box constant. A $12 \times 14$ (totaling 168 variables) 2-D matrix was used to represent the distribution of the source coils. The objective function that needed to be minimized was defined as

$$
F=\left|\sigma_{y}\right|+\sum_{i=1}^{9}\left|B_{x i}\right|
$$

where $B_{x i}$ represents $x$-directional magnetic flux density at a measuring point $i$ [in Fig. 10(b)], and $\sigma_{y}$ is the standard deviation of the $y$-directional flux density at all measuring points.

Both GAs - with and without local optimization - were applied to the topology optimization, and the operating conditions were the same as in Table I. An analytic solution based on

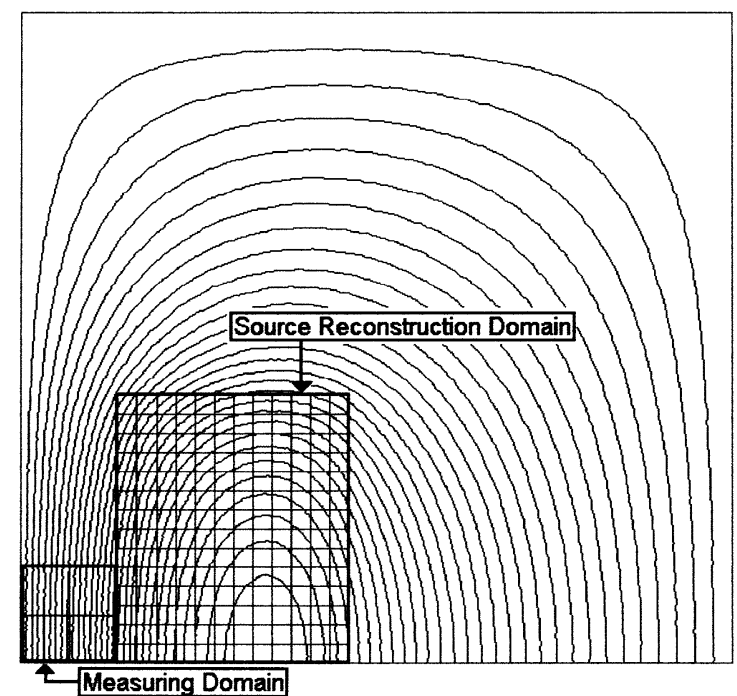

(a)

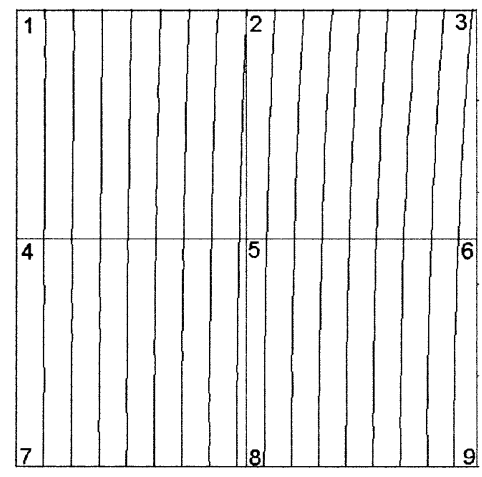

(b)

Fig. 10. Initial magnetic field distribution (a) in whole numerical model and (b) in measuring domain. The FEM was used to illustrate the flux distribution.

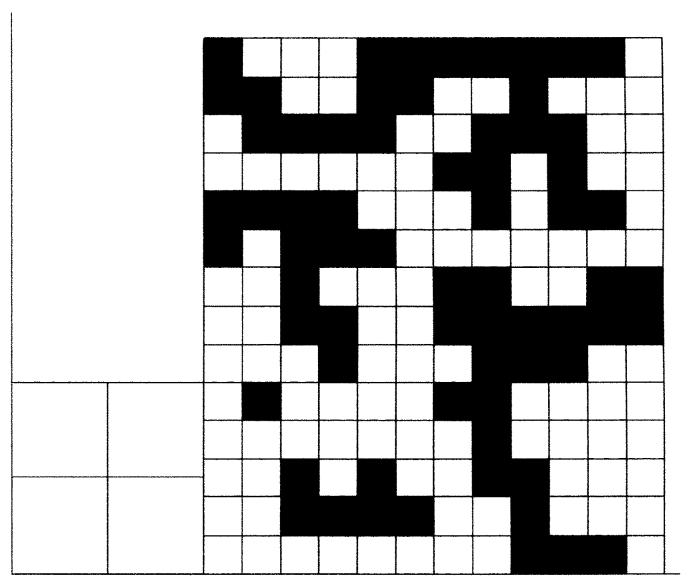

Fig. 11. Best reconstructed solution after 20 repeated executions of GA without local optimization.

Biot-Savart's law was used for the magnetic field calculation. Figs. 11 and 12 show the best reconstructed source distributions after 20 repeated executions of GA with and without local optimization, respectively. Fig. 13 shows the magnetic field distribution in a measuring box by the GA with local optimization. Table III shows the comparison of best, worst, and average values of the objective function (4) after 20 repeated executions. 


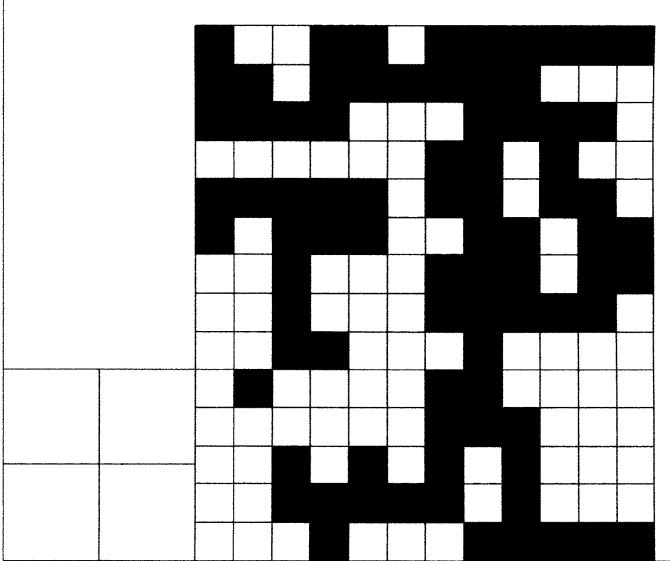

Fig. 12. Best reconstructed solution after 20 repeated executions of GA with local optimization.

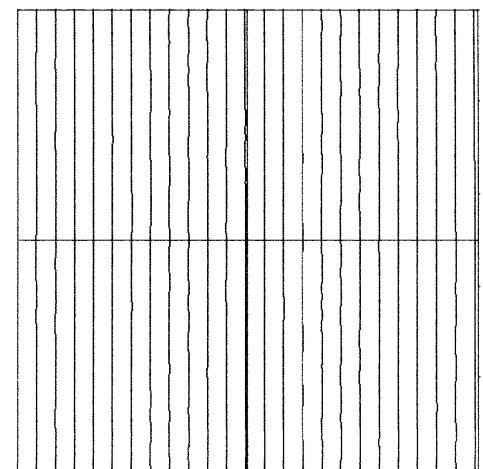

Fig. 13. Final magnetic field distribution in rectangular measuring domain by the GA with local optimization (best solution). FEM was used to illustrate the flux distribution.

TABLE III

COMPARISONS OF CAPABILITY OF GAS WITH AND WITHOUT LOCAL OPTIMIZATION ALGORITHM AFTER 20 REPEATED EXECUTIONS (CASE II)

\begin{tabular}{ccc} 
& Without Local Opt. & With Local Opt. \\
\hline Best Solution $\left[\times 10^{-6} \mathrm{~T}\right]$ & 2.29 & 0.82 \\
Average Solution $\left[\times 10^{-6} \mathrm{~T}\right]$ & 11.54 & 5.81 \\
Worst Solution $\left[\times 10^{-6} \mathrm{~T}\right]$ & 28.77 & 8.43 \\
\hline
\end{tabular}

Smaller value of the solution represents better one as in the previous case.

\section{Case Study III-BLDCM Optimization to Reduce Cogging Torque}

Reduction of cogging torque is a very critical problem in designing permanent-magnet motors including BLDCM [5]. Fig. 14 shows the outer-rotor type BLDCM. To analyze the model, the 2-D finite-element method (FEM), using periodic boundary conditions, was used. The sum of the cogging torques at 11 rotating positions was used for the cost value. The design variables were the material (iron) distribution of the stator poles. Each geometric position in Fig. 15(a) corresponds to the rectangular chromosome matrix in Fig. 15(b). Because the analysis of BLDCM requires extensive calculation time, only a relatively small $7 \times 5$ matrix array was considered. The total population numbered 1000 and the replacement population numbered 600 . The on/off sensitivity method (with the selection probability of 0.8 ) was applied after the 20th iteration. The optimized pole shape is shown in Fig. 16, which yields a

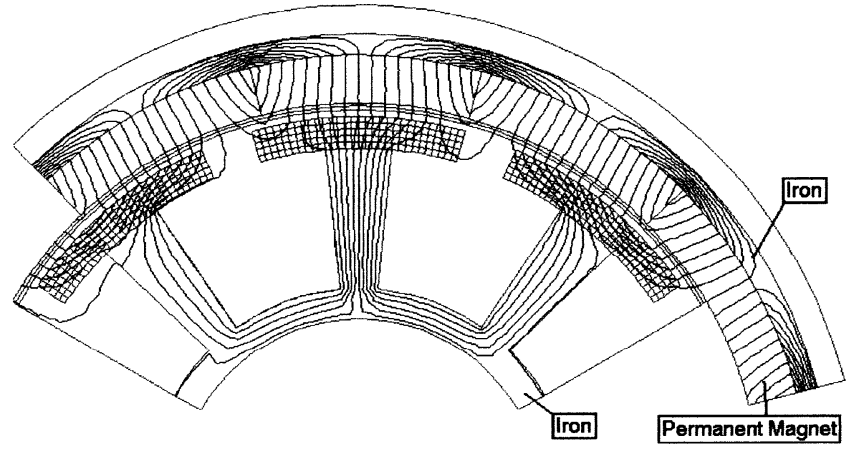

Fig. 14. Numerical model for case study III-BLDCM with outer rotor.

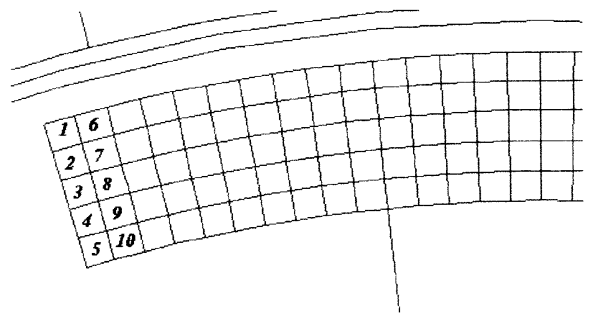

(a)

\begin{tabular}{|c|c|c|c|c|c|c|c|c}
\hline 1 & 6 & & & & & & & \\
\hline 2 & 7 & & & & & & & \\
\hline 3 & 8 & & & & & & &
\end{tabular}$\ldots$

(b)

Fig. 15. Mapping of (a) the real geometry to (b) a rectangular chromosome array- half of one pole is used considering symmetry.

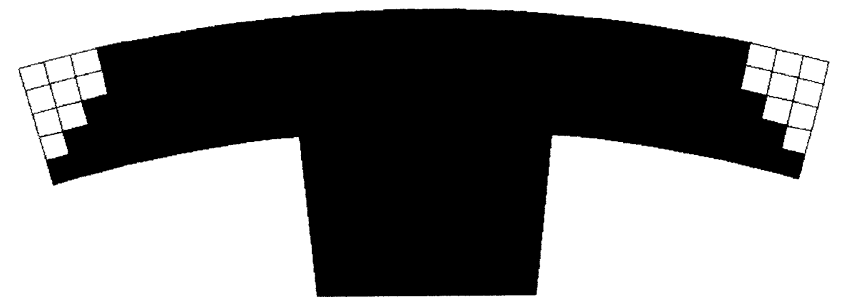

Fig. 16. Best optimized pole shape. A relatively small $7 \times 5$ matrix array was used, considering FEM calculation time.

considerably reduced cogging torque profile as illustrated in Fig. 17. Table IV shows results from 20 repeated executions for the GAs with and without local optimization. Because the number of genes is relatively small compared with the previous two cases, most of both GAs' solutions converged to the optimal pole shape shown in Fig. 16. However, the success rate of the GA with local optimization is higher than that of the GA without local optimization, as shown in the table.

\section{CONCLUSION}

In this paper, a hybrid GA for electromagnetic topology optimization was introduced. A 2-D encoding and geographic crossover was first introduced to electromagnetic topology optimization problems. To improve conventional GA, a novel local optimization algorithm called on/off sensitivity were 


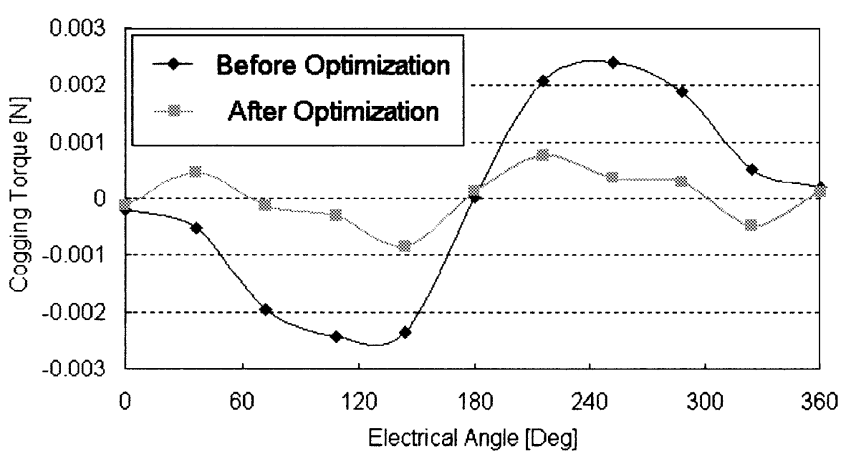

Fig. 17. Variation of cogging torque before and after the pole-shape optimization.

TABLE IV

COMPARISONS OF CAPABILITY OF GAS WITH AND Without LOCAL OPtIMIZATION AlgORITHM AFTER 20 REPEATED EXECUTIONS (CASE III)

\begin{tabular}{ccc}
\hline & Without Local Opt. & With Local Opt. \\
\hline $\begin{array}{c}\text { \# of solutions converged to } \\
\text { the optimal pole shape }\end{array}$ & $\begin{array}{c}14 \\
\text { (out of 20) }\end{array}$ & $\begin{array}{c}19 \\
\text { (out of 20) }\end{array}$ \\
\hline Success rate (\%) & 70 & 95 \\
\hline
\end{tabular}

proposed and hybridized with the existing GA. This proposed algorithm was then successfully applied to various problems, and proved to be a very promising optimization algorithm for use in the field of electromagnetism.

\section{REFERENCES}

[1] J.-K. Kim, D.-H. Cho, H.-K. Jung, and C.-G. Lee, "Niching genetic algorithm adopting restricted competition selection combined with pattern search method," IEEE Trans. Magn., vol. 38, pp. 1001-1004, Mar. 2002.

[2] S. Yang, J. M. Machado, C. Ni, S. L. Ho, and P. Zhou, "A self-learning simulated annealing algorithm for global optimizations of electromagnetic devices," IEEE Trans. Magn., vol. 36, pp. 1004-1008, July 2000.

[3] C.-H. Im, H.-K. Kim, and H.-K. Jung, "Optimization of the coil shape in deflection yoke considering practical coil winding processes," IEEE Trans. Magn., vol. 38, pp. 1077-1080, Mar. 2002.

[4] S. L. Ho, S. Yang, G. Ni, and H. C. Wong, "An improved Tabu search for the global optimizations of electromagnetic devices," IEEE Trans. Magn., vol. 37, pp. 3570-3574, Sept. 2001.

[5] K.-J. Han, H.-S. Cho, D.-H. Cho, and H.-K. Jung, "Optimal core shape design for cogging torque reduction of brushless DC motor using genetic algorithm," IEEE Trans. Magn., vol. 36, pp. 1927-1931, July 2000.

[6] J.-K. Byun, S.-Y. Hahn, and I.-H. Park, "Topology optimization of electrical devices using mutual energy and sensitivity," IEEE Trans. Magn., vol. 35, pp. 3718-3720, Sept. 1999.

[7] J.-K. Byun, J.-H. Lee, I.-H. Park, and S.-Y. Hahn, "Inverse problem application of topology optimization method with mutual energy concept and design sensitivity," IEEE Trans. Magn., vol. 36, pp. 1144-1147, July 2000.

[8] J.-K. Byun, I.-H. Park, and S.-Y. Hahn, "Topology optimization of electrostatic actuator using design sensitivity," IEEE Trans. Magn., vol. 38, pp. 1053-1056, Mar. 2002.

[9] J. P. Cohoon and W. Paris, "Genetic placement," in Proc. IEEE Int. Conf. Computer-Aided Design, 1986, pp. 422-425.
[10] T. N. Bui and B. R. Moon, "On multi-dimensional encoding/crossover," in Proc. 6th Int. Conf. Genetic Algorithms, Pittsburgh, PA, July 1995, pp. 49-56.

[11] B. R. Moon and C. Kim, "A two-dimensional embedding of graphs for genetic algorithms," in Proc. Int. Conf. Genetic Algorithms, 1997, pp. 204-211.

[12] B. R. Moon, Y. S. Lee, and C. K. Kim, "GEORG: VLSI circuit partitioner with a new genetic algorithm framework," J. Intell. Manufact., vol. 9, pp. 401-412, 1998.

[13] J. Holland, Adaptation in Natural and Artificial Systems, 2 ed. Cambridge, MA: MIT Press, 1992.

[14] C. A. Anderson, K. F. Jones, and J. Ryan, "A two-dimensional genetic algorithm for the Ising problem," Complex Syst., vol. 5, pp. 327-333, 1991.

[15] G. Hinton and S. Nowlan, "How learning can guide evolution," Complex Syst., vol. 1, pp. 495-502, 1987.

[16] D. Whitley, V. Gordon, and K. Mathias, "Lamarckian evolution, the Baldwin effect and function optimization," in Proc. Int. Conf. Evolutionary Computation, 1994, pp. 6-15.

[17] A. Dale and M. Sereno, "Improved localization of cortical activity by combining EEG and MEG with MRI cortical surface reconstruction: A linear approach," J. Cogn. Neurosci., pp. 162-176, 1993.

[18] L. Gavit, S. Baillet, J.-F. Mangin, J. Pescatore, and L. Garnero, "A multiresolution framework to MEG/EEG source imaging," IEEE Trans. Biomed. Eng., vol. 48, pp. 1080-1087, Oct. 2001.

[19] J.-H. Lee, J.-K. Byun, K. Choi, and S.-Y. Hahn, "Faster calculation of sensitivity in the source current distribution problem using reciprocity theorem," IEEE Trans. Magn., vol. 37, pp. 3596-3599, Sept. 2001.

[20] D. A. Lowther, W. Mai, and D. N. Dyck, "A comparison of MRI magnet design using a hopfield network and the optimized material distribution method," IEEE Trans. Magn., vol. 34, pp. 2885-2888, Sept. 1998.

Chang-Hwan Im was born in Taegu, Korea, in 1976. He received the B.S. and M.S. degrees in 1999 and 2001, respectively, from Seoul National University, Seoul, Korea, where he is working toward the Ph.D. degree in the School of Electrical Engineering and Computer Science.

His research interests are bioelectromagnetic applications, numerical analysis and design of electromagnetic devices, and microwave passive devices.

Hyun-Kyo Jung (M'85-SM'00) received the B.S., M.S., and Ph.D. degrees from the School of Electrical Engineering, Seoul National University, Seoul, Korea, in 1979, 1981, and 1984, respectively.

He worked as a Member of Faculty at Kangwon National University, Chuncheon, Korea, from 1985 to 1994, and was with Polytechnic University, New York, from 1987 to 1989 . He has been teaching at Seoul National University since 1994. From 1999 to 2000, he also served as a Visiting Professor at the University of California, Berkeley. His research interests cover the various fields of the analysis and design of electric machinery and numerical field analysis of electrical systems, especially with the finite-element method.

Yong-Joo Kim received the B.S. degree from the School of Electrical Engineering, Seoul National University, Seoul, Korea, in 1975, and the M.S. and Ph.D. degrees from Rensselaer Polytechnic Institute, Troy, NY, in 1984 and 1987, respectively.

Since 1979, he has been with the Machine Control and Application Research Group, Korea Electrotechnology Research Institute (KERI), Kyungsangnam-do, Korea. His research interests are analysis and design of high-voltage electrical machines and partial discharge. 